Pacific Journal of Mathematics

SCHLICHT MAPPINGS AND INFINITELY DIVISIBLE 


\title{
SCHLICHT MAPPINGS AND INFINITELY DIVISIBLE KERNELS
}

\author{
ROGER A. HORN
}

The purpose of this note is to give a simple condition which is sufficient for a function on a real interval to be the boundary value of a schlicht (univalent) analytic mapping of the upper half plane into itself. This condition leads to a simple transformation which takes (possibly) non-schlicht mappings into schlicht ones. The methods used have applications to probability theory as well; they yield an interesting class of infinitely divisible characteristic functions.

We shall require some facts about infinitely divisible kernels; for a detailed exposition see [5]. If $I$ is a real interval we denote by $L_{0}(I)$ the set of all continuous complex valued functions which have compact support in $I$ and whose integral over $I$ vanishes. A continuous kernel $K(x, y)$ on $I \times I$ is said to be conditionally positive definite on $I$ if

$$
\iint_{I \times I} K(x, y) \phi(x) \bar{\phi}(y) d x d y \geqq 0
$$

for all functions $\phi \in L_{0}(I)$; it is said to be positive definite on $I$ if (1) is satisfied for all continuous functions $\phi$ with compact support in $I$; it is said to be infinitely divisible on $I$ if (for some fixed continuous determination of the argument) the kernel $K^{\alpha}(x, y)$ is positive definite for all $\alpha>0$.

The connection among these concepts is that a continuous Hermitian kernel $K(x, y)$ with no zeroes is infinitely divisible on $I$ if and only if (for some continuous determination of the argument) the kernel $\log K(x, y)$ is conditionally positive definite on $I$. If $K(x, y)>0$ for all $x, y \in I$ there is, of course, no difficulty about determining the argument. Finally, the relevance of these notions to function theory is indicated by the following result [6], [4]. If $f$ is a differentiable function we define $K_{f}(x, y) \equiv[f(x)-f(y)] /(x-y)$ and agree that $K_{f}(x, x)=f^{\prime}(x)$.

THEOREM 1. Let $f$ be a continuously differentiable real valued function with positive derivative on a real interval $I$. The function $f$ possesses an analytic continuation onto the upper half plane which maps the upper half plane into itself if and only if the kernel $K_{f}(x, y)$ is positive definite on $I$. This mapping is schlicht if and only if $K_{f}(x, y)$ is infinitely divisible on $I$. 
Although this result completely characterizes the boundary values of schlicht mappings, it is in practice much harder to verify that the kernel $K_{f}(x, y)$ is infinitely divisible than to test it for positive definiteness. By our remarks above, one must check whether $\log K_{f}(x, y)$ is conditionally positive definite, but the non-linearity of this expression in $f$ often leads to computational difficulties. In the following, we shall derive a more linear, and hopefully more useful, sufficient condition. Recall that a $C^{\infty}$ function $\phi$ defined on $(0, \infty)$ is completely monotonic if $(-1)^{n} \phi^{(n)}(x) \geqq 0$ for all $x>0$ and all $n=1,2,3, \cdots$.

Lemma 2. Let $H(x, y)$ be a continuous Hermitian kernel on a real interval $I$ such that $\operatorname{Re}\{H(x, y)\}>0$ and such that $-H(x, y)$ is conditionally positive definite. If $\phi$ is any completely monotonic function then the kernel $\phi(H(x, y))$ is positive definite on $I$.

Proof. It is well known that a function $\phi$ is completely monotonic if and only if there exists a nonnegative measure $d \mu$ such that $\phi(x)=\int_{0}^{\infty} e^{-x s} d \mu(s)$ for all $x>0$ ([8], p. 160); in this event $\phi$ is analytic in the whole right half plane. But since $\operatorname{Re}\{H(x, y)\}>0$ and $\exp (-s H(x, y))$ is positive definite (even infinitely divisible) for all $s>0$, it follows that $\phi(H(x, y))=\int_{0}^{\infty} \exp (-s H(x, y)) d \mu(s)$ is convergent and is a positive definite kernel.

An infinitely divisible completely monotonic function $\phi$ is a function such that $\phi^{\alpha}$ is completely monotonic for all $\alpha>0$; if $\phi \not \equiv 0$, a necessary and sufficient condition for this is that the derivative of $-\ln \phi$ be completely monotonic ([3], p. 229). Using the lemma and the definition of an infinitely divisible kernel we obtain

COROLlary 3. Let $\phi$ be a positive differentiable function on $(0, \infty)$ such that $-\phi^{\prime} / \phi$ is completely monotonic, and suppose $H(x, y)$ satisfies the hypotheses of Lemma 2. Then $\phi(H(x, y))$ is an infinitely divisible kernel.

Since the function $\phi(x)=1 / x$ satisfies this condition, the following result is immediate.

CoRollary 4. If $H(x, y)$ satisfies the conditions of Lemma 2, then the kernel $1 / H(x, y)$ is infinitely divisible.

Now suppose that $g$ is a continuously differentiable real valued function on a real interval $I$, so that $K_{g}(x, y)$ is a continuous symmetric kernel. If $g^{\prime}(x)>0$ on $I$ then $K_{g}(x, y)$ is a positive kernel and the 
inverse function $g^{-1}$ is defined on the interval $g(I)$. Thus, if we assume that $-K_{g}(x, y)$ is conditionally positive definite on $I$ then we conclude from Corollary 4 that the kernel

$$
\frac{1}{K_{g}(x, y)}=\frac{x-y}{g(x)-g(y)}=\frac{g^{-1}(g(x))-g^{-1}(g(y))}{g(x)-g(y)}
$$

is infinitely divisible. But this is equivalent to the kernel $K_{g-1}(s, t)$ being infinitely divisible on $g(I)$ and so we may apply Theorem 1 to obtain the conclusion of the following

THEOREm 5. Let $g$ be a continuously differentiable real valued function with positive derivative on a real interval $I$ and suppose that the kernel

$$
-K_{g}(x, y)=-\frac{g(x)-g(y)}{x-y}
$$

is conditionally positive definite on $I$. Then the inverse function $g^{-1}$ has an analytic continuation from $g(I)$ onto the upper half plane which is a schlicht mapping of the upper half plane into itself.

Thus, to ensure that a real function $f$ on a real interval $I$ is the boundary value of a schlicht self-mapping of the upper half plane it is sufficient to check that $f^{\prime}(x)>0$ and that $-K_{f^{-1}}(x, y)$ is conditionally positive definite on $f^{-1}(I)$.

The crucial condition in Theorem 5 is that the kernel $-K_{g}(x, y)$ be conditionally positive definite, and a great deal is known about functions which satisfy this condition. For example, they are real analytic and are analytically continuable onto the upper half plane, they have a simple integral representation, and they arise as the infinitesimal transformations of the pseudo-semigroup $\mathfrak{M}_{\infty}$ of selfmappings of the upper half plane which have real boundary values on $I$ ([6] and [2], pp. 53-54). Furthermore, it is easy to find many non-trivial functions which satisfy this condition. Denote by $\mathfrak{M}_{\infty}(0)$ the class of functions $f$ which are analytic in the upper half plane, map it into itself, are real valued on some open real interval containing zero, and are normalized by the condition $f(0)=0$.

Lemma 6. Let $a$ be a real number, let $b \geqq 0$ and let $f \in \mathfrak{M}_{\infty}(0)$. Then the functions $g_{0}(x)=a, g_{1}(x)=a x, g_{2}(x)=a x^{2}$, and $g_{3}(x)=b x^{2} f(x)$ are such that $K_{g_{i}}(x, y)$ is conditionally positive definite on some neighborhood of the origin, $i=0,1,2,3$.

Proof. This follows from a direct computation for $i=0,1,2$ 
but for $i=3$ we need to know ([1], p. 63) that $f \in \mathfrak{M}_{\infty}(0)$ if and only if

$$
f(x)=\int_{-\varepsilon}^{\varepsilon} \frac{x}{1-t x} d \mu(t)
$$

for some $\varepsilon>0$ and some nonnegative bounded measure $d \mu$ on $[-\varepsilon, \varepsilon]$. Thus, since the assertion for $i=3$ follows for the special case $f(x)=x /(1-t x)$ by direct computation, it follows for all $f \in \mathfrak{M}_{\infty}(0)$ by linearity.

Using the four types of functions introduced in this lemma we can now use Theorem 5 to construct a wide class of schlicht mappings.

THEOREM 7. Let $f \in \mathfrak{M}_{\infty}(0)$, let $a_{1}>0, a_{3} \geqq 0$, and let $a_{0}$ and $a_{2}$ be real numbers. Then the function

$$
g(x)=a_{0}+a_{1} x+a_{2} x^{2}-a_{3} x^{2} f(x)
$$

is such that the inverse function $g^{-1}$ has an analytic continuation from a real neighborhood of $a_{0}$ onto the upper half plane which is a schlicht mapping of the upper half plane into itself.

Proof. The kernel $-K_{g}(x, y)$ is conditionally positive definite by Lemma 6 and $g^{\prime}(x)>0$ in some real neighborhood of zero. The result follows from Theorem 5 .

Although this construction provides a wealth of schlicht mappings, it is far from exhaustive: the functions $f(z)=3[\sqrt[3]{z+1}-1]$ and $f(z)=\log (z+1)$ are schlicht mappings which are not of this form.

REMAEK 1. Linear combinations of the four functions in Lemma 6 are in fact the only smooth functions $g$ such that $K_{g}(x, y)$ is conditionally positive definite. In order to prove this we use the following criterion for a kernel to be conditionally positive definite.

LEMma 8. Let $H(x, y)$ be a continuous kernel on a real interval $I$ and let $x_{0} \in I$. Then $H(x, y)$ is conditionally positive definite on I if and only if the kernel

$$
H_{x_{0}}^{*}(x, y) \equiv H(x, y)-H\left(x, x_{0}\right)-H\left(x_{0}, y\right)+H\left(x_{0}, x_{0}\right)
$$

is positive definite on $I$.

Proof. If $\phi \in L_{0}(I)$, then

$$
\iint_{I \times I} H_{x_{0}}^{*}(x, y) \phi(x) \bar{\phi}(y) d x d y=\iint_{I \times I} H(x, y) \phi(x) \bar{\phi}(y) d x d y,
$$


and hence $H(x, y)$ is conditionally positive definite if $H_{x_{0}}^{*}(x, y)$ is positive definite. Conversely, suppose $H(x, y)$ is conditionally positive definite and let $\left\{f_{n}(x)\right\}, n=1,2,3, \cdots$ be an approximate identity based at $x_{0}$, i.e., each $f_{n}$ is a nonnegative continuous function with support in $I \cap\left[x_{0}-n^{-1}, x_{0}+n^{-1}\right]$ and $\int_{I} f_{n}(x) d x=1$ for all $n$. If $\phi$ is any continuous function with compact support in $I$, let $\phi_{n}(x) \equiv \phi(x)-$ $f_{n}(x) \int_{I} \phi(t) d t$ and observe that $\phi_{n} \in L_{0}(I)$ for all large $n$. Thus,

$$
\begin{aligned}
0 \leqq & \iint_{I \times I} H(x, y) \dot{\phi}_{n}(x) \bar{\phi}_{n}(y) d x d y \\
= & \iint_{I \times I}\{H(x, y) \\
& \quad-\int_{I} H(x, t) f_{n}(t) d t-\int_{I} H(s, y) f_{n}(s) d s \\
& \left.\quad+\iint_{I \times I} H(s, t) f_{n}(s) f_{n}(t) d s d t\right\} \dot{\phi}(x) \bar{\phi}(y) d x d y \\
& \rightarrow \iint_{I \times I}\left\{H(x, y)-H\left(x, x_{0}\right)-H\left(x_{0}, y\right)+H\left(x_{0}, x_{0}\right)\right\} \phi(x) \bar{\phi}(y) d x d y \\
= & \iint_{I \times I} H_{x_{0}}^{*}(x, y) \phi(x) \bar{\phi}(y) d x d y
\end{aligned}
$$

as $n \rightarrow \infty$. Since $\phi$ is arbitrary, we conclude that the kernel $H_{x_{0}}^{*}(x, y)$ must be positive definite.

Lemma 9. Let $K(x, y)$ be a continuous kernel on a real interval I. Then $x y K(x, y)$ is positive definite kernel if and only if $K(x, y)$ is a positive definite kernel.

Proof. If zero is not a point of $I$ this is trivial, so suppose $0 \in I$, let $\varepsilon>0$, and denote by $f_{\varepsilon}$ the unique even function such that

$$
f_{\varepsilon}(x) \equiv\left\{\begin{array}{l}
0 \text { if } x \in[0, \varepsilon] \\
\varepsilon^{-1}(x-\varepsilon) \text { if } x \in[\varepsilon, 2 \varepsilon] \\
1 \text { if } x \geqq \varepsilon .
\end{array}\right.
$$

Let $M \equiv \sup _{I \times I}|K(x, y)|$, let $\phi$ be a continuous function with compact support in $I$, and assume that $x y K(x, y)$ is positive definite on $I$. Then

$$
\begin{aligned}
& \iint_{I \times I} K(x, y) \phi(x) \bar{\phi}(y) d x d y \\
= & \iint_{I \times I} K(x, y) \phi(x) \bar{\phi}(y)\left(1-f_{\varepsilon}(y)\right) d x d y \\
& +\iint_{I \times I} K(x, y) \phi(x) \bar{\phi}(y) f_{\varepsilon}(y)\left(1-f_{\varepsilon}(x)\right) d x d y \\
& +\iint_{I \times I} K(x, y) \phi(x) f_{\varepsilon}(x) \bar{\phi}(y) f_{\varepsilon}(y) d x d y
\end{aligned}
$$




$$
\begin{aligned}
\geqq & -\iint_{I \times I}\left|K(x, y) \phi(x) \bar{\phi}(y)\left(1-f_{\varepsilon}(y)\right)\right| d x d y \\
& -\iint_{I \times I}\left|K(x, y) \bar{\phi}(y) f_{\varepsilon}(y) \dot{\phi}(x)\left(1-f_{\varepsilon}(x)\right)\right| d x d y \\
& +\iint_{I \times I} x y K(x, y) x^{-1} \phi(x) f_{\varepsilon}(x) y^{-1} \bar{\phi}(y) f_{c}(y) d x d y \\
\geqq & -6 M \varepsilon \sup _{I}|\dot{\phi}(x)| \int_{I}|\phi(x)| d x .
\end{aligned}
$$

For the last inequality we have used the hypothesis that $x y K(x, y)$ is positive definite and the fact that the function $x^{-1} \dot{\phi}(x) f_{\varepsilon}(x)$ is a continuous function with compact support in $I$. Since $\varepsilon>0$ is arbitrary we conclude that

$$
\iint_{I \times I} K(x, y) \dot{\phi}(x) \bar{\phi}(y) d x d y \geqq 0,
$$

i.e., $K(x, y)$ is positive definite. The converse is trivial.

Now assume that the kernel $H(x, y)$ is of the special form $H(x, y)=K_{g}(x, y)$, where $g$ is a real valued function which is three times continuously differentiable on an open real interval containing zero. Assume that $g(0)=g^{\prime}(0)=g^{\prime \prime}(0)=0$. Then $g(x) / x^{2}$ is continuously differentiable and

$$
\begin{aligned}
& H_{v}^{*}(x, y)=K_{g}(x, y)-K_{g}(x, 0)-K_{g}(0, y)+K_{g}(0,0) \\
= & x y \frac{\frac{g(x)}{x^{2}}-\frac{g(y)}{y^{2}}}{x-y}=x y K_{h}(x, y),
\end{aligned}
$$

where we set $h(x) \equiv g(x) / x^{2}$. Thus, Lemma 8 says that $K_{g}(x, y)$ is conditionally positive definite if and only if $x y K_{h}(x, y)$ is positive definite, and Lemma 9 says this is equivalent to the kernel $K_{h}(x, y)$ being positive definite. We conclude that $K_{g}(x, y)$ is conditionally positive definite if and only if $K_{h}(x, y)$ is positive definite. But this means that $h(x)=g(x) / x^{2} \in \mathfrak{M}_{\infty}(0)$ and hence $h$ has the integral representation (2). The normalization we assumed for $g$ can always be attained by subtracting a suitable quadratic polynomial, since Lemma 6 shows that every such polynomial has a conditionally positive definite difference quotient kernel. We summarize our results as

THEOREM 10. Let $g$ be real valued function on an open real interval I containing zero. The following are equivalent:

(a) The function $g$ is three times continuously differentiable and the kernel $K_{g}(x, y)=[g(x)-g(y)] /(x-y)$ is conditionally positive 
definite on $I$.

(b) The function $g$ has the form

$$
g(x)=a_{0}+a_{1} x+a_{2} x^{2}+a_{3} x^{2} f(x),
$$

where $a_{0}, a_{1}, a_{2}$ are real numbers, $a_{3} \geqq 0$ and $f \in \mathfrak{M}_{\infty}(0)$.

(c) The function $g$ has the form

$$
g(x)=a_{0}+a_{1}+a_{2} x^{2}+\int_{-\varepsilon}^{\varepsilon} \frac{x^{3} d \mu}{1-x t}
$$

where $a_{0}, a_{1}, a_{2}$ are real numbers, $\varepsilon \geqq 0$ and $d \mu$ is a nonnegative bounded measure.

It should be noted that it is sufficient in (a) to assume only that $g$ is continuously differentiable; the condition on the kernel then implies that $g$ is analytic [6]. This characterization of the functions $g$ such that $K_{g}(x, y)$ is conditionally positive definite was obtained first by C. FitzGerald [2] using less elementary results on analytic kernels.

REMARK 2. Lemma 2 and its corollaries are also useful in probability theory where one is interested in continuous Hermitian kernels of the form $K(x, y)=f(x-y), f(0)=1$. Such a kernel is positive definite if and only if $f(x)$ is the Fourier transform of a (unique) probability measure on the line, i.e., $f(x)$ is a characteristic function; this kernel is infinitely divisible if and only if the measure is infinitely divisible. If $f(x)$ is the characteristic function of an infinitely divisible probability measure, then the kernel $H(x, y)=$ $\ln f(x-y)$ is conditionally positive definite and has nonpositive real part since $|f(x)| \leqq 1$ for all real $x$. Thus, the kernel $H(x, y)=\lambda-\ln f(x-y)$ satisfies the hypotheses of Lemma 2 if $\lambda>0$ and hence the kernel

$$
\frac{\lambda}{\lambda-\ln f(x-y)}
$$

is infinitely divisible by Corollary 4 . But this means that the function $\phi(x)=\lambda /(\lambda-\ln f(x))$ is an infinitely divisible characteristic function whenever $f$ is an infinitely divisible characteristic function and $\lambda>0$. This result was obtained by F. W. Steutel [7] from very different considerations.

\section{REFERENCES}

1. J. Bendat and S. Sherman, Monotone and convex matrix functions, Trans. Amer. Math. Soc., 79 (1965), 58-71. 
2. C. FitzGerald, Topics in Geometric Function Theory, Stanford University PhD Thesis, 1967.

3. R. A. Horn, On infinitely divisible matrices, kernels, and functions, Z. Wahrscheinlichkeitstheorie verw. Geb., 8 (1967), 219-230.

4. - On boundary values of a Schlicht mapping, Proc. Amer. Math. Soc., 18 (1967), 782-787.

5. - The theory of infinitely divisible matrices and kernels, Trans. Amer. Math. Soc., 136 (1969), 269-286.

6. C. Loewner, On Schlicht-monotonic functions of higher order, J. Math. Anal. Appl., 14 (1966), 320-325.

7. F. W. Steutel, A class of infinitely divisible mixtures, Ann. Math. Stat., 39 (1968), 1153-1157.

8. D. V. Widder, The Laplace Transform, Princeton University Press, Princeton, N. J., 1946.

Received August 20, 1970. This work was partially supported by the National Science Foundation with Grant GP-13258 and by the Deutscher Akademischer Austauschdienst with a grant at the Mathematisches Institut der Universität Marburg.

The Johns Hopkins University

Present address: University of MARYLAND 


\section{PACIFIC JOURNAL OF MATHEMATICS}

\section{EDITORS}

\author{
H. SAMELSON \\ Stanford University \\ Stanford, California 94305 \\ C. R. HовBY \\ University of Washington \\ Seattle, Washington 98105
}

J. DUGUndis

Department of Mathematics

University of Southern California

Los Angeles, California 90007

RICHARD ARENS

University of California

Los Angeles, California 90024

\section{ASSOCIATE EDITORS}
E. F. BeCKenbaCH
B. H. NeUmanN
F. WOLF
K. YoshidA

\section{SUPPORTING INSTITUTIONS}

\author{
UNIVERSITY OF BRITISH COLUMBIA \\ CALIFORNIA INSTITUTE OF TECHNOLOGY \\ UNIVERSITY OF CALIFORNIA \\ MONTANA STATE UNIVERSITY \\ UNIVERSITY OF NEVADA \\ NEW MEXICO STATE UNIVERSITY \\ OREGON STATE UNIVERSITY \\ UNIVERSITY OF OREGON \\ OSAKA UNIVERSITY \\ UNIVERSITY OF SOUTHERN CALIFORNIA
}

\author{
STANFORD UNIVERSITY \\ UNIVERSITY OF TOKYO \\ UNIVERSITY OF UTAH \\ WASHINGTON STATE UNIVERSITY \\ UNIVERSITY OF WASHINGTON

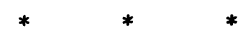 \\ AMERICAN MATHEMATICAL SOCIETY \\ CHEVRON RESEARCH CORPORATION \\ NAVAL WEAPONS CENTER
}

The Supporting Institutions listed above contribute to the cost of publication of this Journal, but they are not owners or publishers and have no responsibility for its content or policies.

Mathematical papers intended for publication in the Pacific Journal of Mathematics should be in typed form or offset-reproduced, (not dittoed), double spaced with large margins. Underline Greek letters in red, German in green, and script in blue. The first paragraph or two must be capable of being used separately as a synopsis of the entire paper. The editorial "we" must not be used in the synopsis, and items of the bibliography should not be cited there unless absolutely necessary, in which case they must be identified by author and Journal, rather than by item number. Manuscripts, in duplicate if possible, may be sent to any one of the four editors. Please classify according to the scheme of Math. Rev. Index to Vol. 39. All other communications to the editors should be addressed to the managing editor, Richard Arens, University of California, Los Angeles, California, 90024.

50 reprints are provided free for each article; additional copies may be obtained at cost in multiples of 50 .

The Pacific Journal of Mathematics is published monthly. Effective with Volume 16 the price per volume (3 numbers) is $\$ 8.00$; single issues, $\$ 3.00$. Special price for current issues to individual faculty members of supporting institutions and to individual members of the American Mathematical Society: $\$ 4.00$ per volume; single issues $\$ 1.50$. Back numbers are available.

Subscriptions, orders for back numbers, and changes of address should be sent to Pacific Journal of Mathematics, 103 Highland Boulevard, Berkeley, California, 94708.

PUBLISHED BY PACIFIC JOURNAL OF MATHEMATICS, A NON-PROFIT CORPORATION

Printed at Kokusai Bunken Insatsusha (International Academic Printing Co., Ltd.), 7-17, Fujimi 2-chome, Chiyoda-ku, Tokyo, Japan. 


\section{Pacific Journal of Mathematics}

Vol. 38, No. $2 \quad$ April, 1971

Richard Davis Anderson and Thomas Ashland Chapman, Extending

homeomorphisms to Hilbert cube manifolds .................. 281

Nguyen Huu Anh, Restriction of the principal series of $\operatorname{SL}(n, \mathbf{C})$ to some

reductive subgroups................................ 295

David W. Boyd, Indices for the Orlicz spaces . . . . . . . . . . . . 315

William Garfield Bridges, The polynomial of a non-regular digraph ...... 325

Billie Chandler Carlson, Robert K. Meany and Stuart Alan Nelson, Mixed

arithmetic and geometric means........................ 343

H. A. Çelik, Commutative associative rings and anti-flexible rings ...... 351

Hsin Chu, On the structure of almost periodic transformation groups ...... 359

David Allyn Drake, The translation groups of n-uniform translation

Hjelmslev planes ................................ 365

Michael Benton Freeman, The polynomial hull of a thin two-manifold . . . . 377

Anthony Alfred Gioia and Donald Goldsmith, Convolutions of arithmetic

functions over cohesive basic sequences .................... 391

Leslie C. Glaser, A proof of the most general polyhedral Schoenflies

conjecture possible ................................

Thomas Lee Hayden and Ted Joe Suffridge, Biholomorphic maps in Hilbert

space have a fixed point ................................ 419

Roger Alan Horn, Schlicht mappings and infinitely divisible kernels ...... 423

Norman Ray Howes, On completeness ...................... 431

Hideo Imai, Sario potentials on Riemannian spaces................ 441

A. A. Iskander, Subalgebra systems of powers of partial universal

algebras.

Barry E. Johnson, Norms of derivations of $\mathscr{L}(\mathrm{X})$.

David Clifford Kay and Eugene W. Womble, Axiomatic convexity theory and relationships between the Carathéodory, Helly, and Radon numbers

Constantine G. Lascarides, A study of certain sequence spaces of Maddox

and a generalization of a theorem of Iyer .............

C. N. Linden, On Blaschke products of restricted growth .

John S. Lowndes, Some triple integral equations ................. 515

Declan McCartan, Bicontinuous preordered topological spaces ......... 523

S. Moedomo and J. Jerry Uhl, Jr., Radon-Nikodým theorems for the Bochner and Pettis integrals ...

Calvin Cooper Moore and Joseph Albert Wolf, Totally real representations

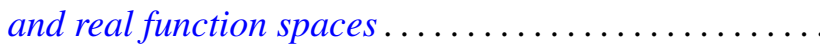

Reese Trego Prosser, A form of the moment problem for Lie groups. ... 